\title{
Gene transfer: anything goes in plant mitochondria
}

\author{
John M Archibald*1 and Thomas A Richards² \\ See research article: http://www.biomedcentral.com/1741-7007/8/150
}

\begin{abstract}
Parasitic plants and their hosts have proven remarkably adept at exchanging fragments of mitochondrial DNA. Two recent studies provide important mechanistic insights into the pattern, process and consequences of horizontal gene transfer, demonstrating that genes can be transferred in large chunks and that gene conversion between foreign and native genes leads to intragenic mosaicism. A model involving duplicative horizontal gene transfer and differential gene conversion is proposed as a hitherto unrecognized source of genetic diversity.
\end{abstract}

\section{Horizontal gene transfer in plants}

Among the most profound biological insights gleaned from the past decade of comparative genomics has been the realization that horizontal gene transfer (HGT) has impacted the genetic make up of virtually everything we have chosen to sequence. Long recognized as a major force in the evolution of prokaryotic genomes [1] and somewhat more recently for microbial eukaryotes [2], HGT can also impact the genomes of complex, multicellular organisms: case in point, plant mitochondrial DNA (mtDNA). Parasitic plants and their hosts (Figure 1) have proven to be avid donors and recipients of mtDNA [3], and two recent studies, one in the pages of $B M C$ Biology [4], have provided new mechanistic detail on the causes and consequences of plant HGT [4,5]. HGTs involving plant nuclear and chloroplast DNA appear (for now at least) to be rare [6,7]. In contrast, plant mtDNA appears to be highly mobile, a fact that has significant practical and theoretical implications for plant biology.

The first suggestions that plant mitochondria might be exceptional in terms of DNA uptake came from studies of

\footnotetext{
*Correspondence: john.archibald@dal.ca

${ }^{1}$ Canadian Institute for Advanced Research, Program in Integrated Microbial Biodiversity, Department of Biochemistry and Molecular Biology, Sir Charles Tupper Medical Building, Dalhousie University, Halifax, NS B3H 1X5, Canada Full list of author information is available at the end of the article
}

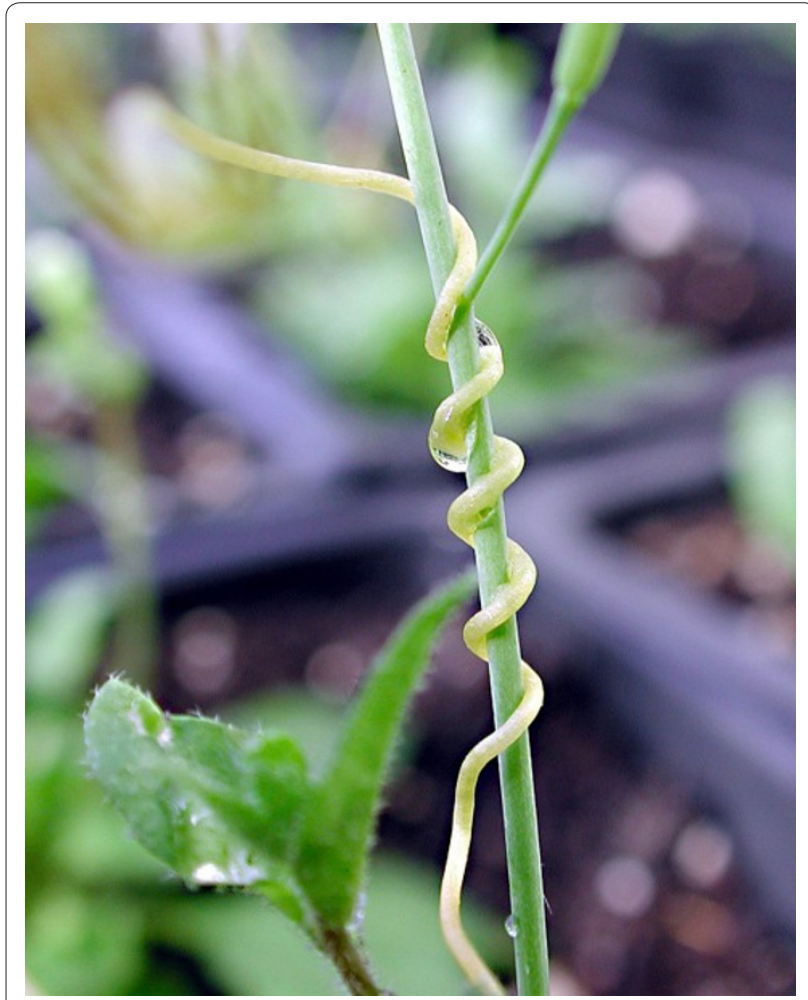

Figure 1. The parasitic plant Cuscuta wrapping around one of its many possible hosts, Arabidopsis. Image kindly provided by Dr Collin Purrington, Swarthmore College.

their fungal-derived, homing group I introns (for example, [8]), and a growing body of evidence for plantto-plant HGT has since accumulated [3]. The current champion is Amborella trichopoda, a 'primitive' flowering plant found exclusively on the island of New Caledonia, the mtDNA of which is littered with foreign genes acquired from both angiosperm and non-angiosperm donors $[9,10]$.

How exactly does plant HGT happen? An important clue comes from the fact that while examples of ancient and 'recent' HGT events involving chloroplast DNA have been documented [2], such events appear to be very infrequent [6]. This makes sense given that plant 
mitochondria possess active DNA uptake systems and are capable of fusion; chloroplasts do not and are not (see [3] and references therein). Thus, given direct physical contact between host and parasite tissue, ample opportunity for mtDNA uptake and exchange would seem to exist. Yet despite extensive phylogenetic evidence supporting the notion that plant mitochondrial HGT is rampant, numerous mechanistic uncertainties remain. These include the question of whether DNA or RNA is the donor molecule and whether a virus or some other vector mediates the transfer.

\section{Gene transfer, gene conversion, and intragenic mosaicism}

Mower et al. [4] sought to elucidate the process of plantto-plant HGT by using PCR to survey mitochondrial genes in species of the parasitic plant Cuscuta (Figure 1) and one of its many hosts, the dicot weed Plantago. After initially casting a wide net to capture ten protein and RNA genes from both Cuscuta gronovii and Plantago coronopus mtDNA, they sequenced three genes, atp1, atp 6 and matR, from a range of host and parasite relatives and showed that each gene appears to have been transferred recently (within the last few million years) from the mitochondrial genome of Cuscuta to that of Plantago. In and of itself this is no longer surprising, but the authors demonstrate that (i) the three genes appear to have been transferred together in the context of a relatively large fragment of DNA (and not RNA, which can be inferred due to the presence of unedited cytidine residues at sites known to undergo RNA editing); (ii) both native and 'foreign' homologs (xenologs), the latter in the form of pseudogenes, co-exist in several species; and (iii) multiple gene conversion events have occurred between co-resident loci.

Hao et al. [5] have gone even further. They uncovered a 'gorgeous mosaic' of multiple mitochondrial genes and gene fragments in various plant host-parasite lineages, including a striking chloroplast-to-mitochondrion transfer involving a region of atp1. In total, approximately onethird of the HGT events investigated (5 of 17 genes) appear to have involved at least some gene conversion the non-reciprocal exchange of DNA between homologous sequences - suggesting that this process plays an important role in the integration of foreign genetic material. The true significance of HGT-associated gene conversion may in fact be underestimated because of differential gene loss, lack of phylogenetic resolution and insufficiently sensitive detection methods. The authors propose a model - duplicative HGT and differential gene conversion (DH-DC) - in which intra- and interorganellar gene transfer and recombination are creative forces in the generation of mitochondrial genetic diversity.
By duplicative HGT, Hao et al. mean the transfer and integration of a foreign set of genes, a single gene, or a gene fragment from donor to recipient mtDNA that does not instantly replace its endogenous counterpart. Herein lies the key to the model, as the co-existence, however transient, of foreign and native loci within the same subcellular compartment allows gene conversion to occur. Gene conversion is a well-understood process (for example, as a generator of allelic diversity during meiosis), and in the context of DH-DC, gene conversion, occurring in either a continuous or discontinuous manner, gives rise to patchwork recombinants. Such heterogeneity is not phenotypically 'silent': the recombinant atp 1 and mat $R$ genes uncovered by Hao et al. yield proteins with different amino acid sequences [5].

\section{DH-DC in plant mtDNA: impact and implications}

The results of Mower et al. [4] and Hao et al. [5] are both troubling and satisfying. Troubling because recombination between resident and foreign gene copies, no matter how transient the latter, has the potential to 'wreak havoc' on the results of even the most cautious phylogeneticist intent on inferring organismal phylogenies from plant mitochondrial genes [5]. In addition, mutation rate estimates for plant mtDNA - painstakingly obtained and long considered to be exceptionally low [3,11] - should, in the face of the DH-DC model, now be considered overestimates: a certain fraction of single-nucleotide differences observed between sequences will be due to gene conversion, not point mutation. Ultimately, though, satisfaction comes from a deeper understanding and appreciation of the true complexity of plant mtDNA evolution.

More generally, Hao et al. [5] raise the intriguing possibility that DH-DC could be a driver of gene evolution in prokaryotes, one that has thus far gone undetected. And what of nuclear genomes? In concluding their study of Plantago-Cuscuta HGT, Mower et al. [4] state that '...unravelling this history will probably require sequencing multiple mitochondrial and nuclear genomes from Plantago.' Given the pace at which sequencing technologies are evolving, it is hard to imagine this not happening in the near future, not just within plants but also for microbes and multicellular organisms across the full breadth of eukaryotic diversity. With the exception of specific lineages such as fungi, whose nuclear genomes are being sequenced at both shallow and deep evolutionary divergences, the field of comparative genomics is still in 'gap filling' mode. The Hao et al. [5] and Mower et al. [4] studies underscore the fact that when it comes to HGT the devil is in the details: only in the context of meticulous comparisons of both closely and distantly related genomes is a deep understanding of the pattern, process and full scope of eukaryotic HGT likely to emerge. 


\section{Author details}

'Canadian Institute for Advanced Research, Program in Integrated Microbial Biodiversity, Department of Biochemistry and Molecular Biology, Sir Charles Tupper Medical Building, Dalhousie University, Halifax, NS B3H 1X5, Canada.

${ }^{2}$ Department of Zoology, Natural History Museum London, Cromwell Road, London, SW7 5BD, UK.

Published: 22 December 2010

\section{References}

1. Ochman H, Lawrence JG, Groisman EA: Lateral gene transfer and the nature of bacterial innovation. Nature 2000, 405:299-304.

2. Keeling PJ, Palmer JD: Horizontal gene transfer in eukaryotic evolution. Nat Rev Genet 2008, 9:605-618.

3. Richardson AO, Palmer JD: Horizontal gene transfer in plants. J Exp Bot 2007, 58:1-9.

4. Mower JP, Stefanovic S, Hao W, Gummow JS, Jain K, Ahmed D, Palmer JD: Horizontal acquisition of multiple mitochondrial genes from a parasitic plant followed by gene conversion with host mitochondrial genes. BMC $\mathrm{Bio} / 2010,8: 150$

5. Hao W, Richardson AO, Zheng Y, Palmer JD: Gorgeous mosaic of mitochondrial genes created by horizontal transfer and gene conversion. Proc Natl Acad Sci USA 2010, [www.pnas.org/cgi/doi/10.1073/ pnas.1016295107].

6. Rice DW, Palmer JD: An exceptional horizontal gene transfer in plastids: gene replacement by a distant bacterial paralog and evidence that haptophyte and cryptophyte plastids are sisters. BMC Biol 2006, 4:31.

7. Richards TA, Soanes DM, Foster PG, Leonard G, Thornton CR, Talbot NJ: Phylogenomic analysis demonstrates a pattern of rare and ancient horizontal gene transfer between plants and fungi. Plant Cell 2009, 21:1897-1911.

8. Cho Y, Qiu YL, Kuhlman P, Palmer JD: Explosive invasion of plant mitochondria by a group I intron. Proc Natl Acad Sci USA 1998, 95:14244-14249.

9. Bergthorsson U, Adams KL, Thomason B, Palmer JD: Widespread horizontal transfer of mitochondrial genes in flowering plants. Nature 2003, 424:197-201.

10. Bergthorsson U, Richardson AO, Young GJ, Goertzen LR, Palmer JD: Massive horizontal transfer of mitochondrial genes from diverse land plant donors to the basal angiosperm Amborella. Proc Natl Acad Sci USA 2004, 101:17747-17752.

11. Wolfe KH, Li WH, Sharp PM: Rates of nucleotide substitution vary greatly among plant mitochondrial, chloroplast, and nuclear DNAs. Proc Natl Acad Sci USA 1987, 84:9054-9058.

doi:10.1186/1741-7007-8-147

Cite this article as: Archibald JM, Richards TA: Gene transfer: anything goes in plant mitochondria. BMC Biology 2010, 8:147. 